\title{
High-frequency asymptotics for the modified Helmholtz equation in a half-plane
}

Min-Hai Huang*

\section{*Correspondence:}

hmh9520@sina.com

College of Mathematics and Information Sciences, Zhaoging

University, Zhaoqing, GuangDong

526061, China

\begin{abstract}
Based on the integral representations of the solution derived via Fokas' transform method, the high-frequency asymptotics for the solution of the modified Helmholtz equation, in a half-plane and subject to the Dirichlet condition, is discussed. For the case of piecewise constant boundary data, full asymptotic expansions of the solution are obtained by using Watson's lemma and the method of steepest descents for definite integrals.
\end{abstract}

MSC: 35B40; 35C15; 35J05; 41A60

Keywords: high-frequency asymptotics; Fokas' transform method; method of steepest descents; modified Helmholtz equation; Dirichlet boundary value problem

\section{Introduction}

There is huge mathematical and engineering interest in acoustic and electromagnetic wave scattering problems, driven by many applications such as modeling radar, sonar, acoustic noise barriers, atmospheric particle scattering, ultrasound, and VLSI [1]. Many problems of scattering of time-harmonic acoustic or electromagnetic waves can be formulated as the Helmholtz and modified Helmholtz equations, supplemented with appropriate boundary conditions. Many efforts have been made to develop efficient numerical schemes and approximate methods to deal with the problems of high wavenumbers (i.e., high frequencies) $[2-6]$. It is noted in $[4,7]$ that a question yet to be fully resolved is to obtain accurate approximations of the solutions with a reasonable computational cost in the high-frequency case. Therefore it seems desirable, and difficult, to consider the high-frequency asymptotics of the equation and its modified version. This is the main motive of the present investigation. Applying the theory of asymptotic analysis [8-10], one may achieve a high degree of accuracy with only a few leading terms in the asymptotic expansions of the solution involved.

The objective of the present paper is to consider the following Dirichlet boundary value problem of the modified Helmholtz equation in the upper half-plane $\Omega$ :

$$
\begin{aligned}
& \triangle q(z, \bar{z})-4 \beta^{2} q(z, \bar{z})=0, \quad z \in \Omega, \\
& q=d(z), \quad z \in \Gamma
\end{aligned}
$$

where $\mathbf{n}$ is the outer normal vector, $\Delta=\frac{\partial^{2}}{\partial x^{2}}+\frac{\partial^{2}}{\partial y^{2}}=4 \frac{\partial^{2}}{\partial z \partial \bar{z}}$ is the usual Laplace operator, $z=x+i y$ and $\bar{z}=x-i y, \Gamma=\partial \Omega$ is the closed real line, $d$ decays sufficiently fast at infinity (e.g. $\left.d \in L^{1}\left(\mathbb{R}^{+}\right) \cap L^{2}\left(\mathbb{R}^{+}\right)\right)$.

@2014 Huang; licensee Springer. This is an Open Access article distributed under the terms of the Creative Commons Attribution License (http://creativecommons.org/licenses/by/2.0), which permits unrestricted use, distribution, and reproduction in any medium, provided the original work is properly cited. 
As a first step, we given the integral representation for the solution of the Dirichlet boundary value problem (1.1)-(1.2) for general $d(z)$ derived by Fokas' transform method.

And then, by using Watson's lemma and the method of steepest descents for definite integrals, we focus on the high-frequency asymptotics with respect to specific Dirichlet data, namely,

$$
q=d(z):= \begin{cases}D, & z \in[a, b] \\ 0, & z \in \Gamma \backslash[a, b]\end{cases}
$$

where $[a, b]$ refers to a finite interval and $D$ is an arbitrary constant.

\section{The integral representation for the solution}

For the Dirichlet boundary value problem (1.1)-(1.2), by using Fokas' transform method, we have the following lemma.

Lemma 1 Assume that the function $q(x, y)$ solves the modified Helmholtz equation (1.1) in the upper half z-plane $\Omega$, and that it satisfies the Dirichlet boundary conditions (1.2), then the integral representation is valid:

$$
q=\frac{\beta}{2 \pi} \int_{0}^{\infty} e^{i \beta\left(k z-\frac{\bar{z}}{k}\right)}\left(k+\frac{1}{k}\right) D(k) \frac{d k}{k},
$$

where

$$
D(k)=\int_{-\infty}^{\infty} e^{-i \beta(k-1 / k) s} d(s) d s
$$

The interested reader is referred to [11, Ch.11] and [12] for derived in detail. Accordingly, when we specify (1.2) in accordance to the piecewise constant Dirichlet data (1.3), we have the following lemma.

Lemma 2 Assume that the function $q(x, y)$ solves the modified Helmholtz equation (1.1) in the upper half z-plane $\Omega$, and that it satisfies the Dirichlet boundary conditions (1.3), then the integral representation is valid:

$$
q(x, y)=\frac{D}{2 \pi i} \int_{l} e^{i \beta\left(k z-\frac{\bar{z}}{k}\right)}\left[e^{i \beta a\left(\frac{1}{k}-k\right)}-e^{i \beta b\left(\frac{1}{k}-k\right)}\right] \frac{k^{2}+1}{k\left(k^{2}-1\right)} d k,
$$

where $l$ is an arbitrary ray in the first quadrant of the complex $k$-plane, oriented from the origin to infinity, with an angle between $l$ and the positive real axis less than $\pi-\arg (z-b)$.

\section{Asymptotic approximations}

Our goal in this section is to study the asymptotical behavior of the solution to the Dirichlet problem of the modified Helmholtz equation, as the frequency, or, equivalently, the wavenumber, approaches infinity. This large- $\beta$ asymptotic analysis will be based on the integral representation (2.3), and it will be carried out by using the method of steepest descents. 
Figure 1 The steepest descent path $\Gamma$.

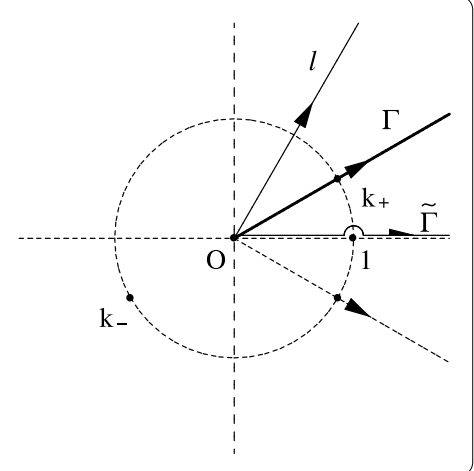

First we note that (2.3) can be expressed as the sum of two integrals of the form

$$
I(\beta)=\frac{D}{2 \pi i} \int_{l} e^{i \beta\left[k(z+A)-\frac{1}{k} \overline{(z+A)]}\right.} \frac{k^{2}+1}{k\left(k^{2}-1\right)} d k,
$$

where $A=-a,-b$, and the path $l$, the same as in (2.3), is initially chosen as a ray in the first quadrant, emanating from the $k$-origin, with an open angle with the positive real line not exceeding $\pi-\arg (z-b)$. We denote

$$
\varphi(k)=i\left[k(z+A)-\frac{1}{k} \overline{(z+A)}\right], \quad k=u+i v, z=x+i y, z+A=r e^{i \theta} .
$$

The phase function $\varphi(k)$ has a pair of saddle points $k_{ \pm}= \pm i e^{-i \theta}= \pm e^{i(\pi / 2-\theta)}$ determined by $\varphi^{\prime}(k)=0$, lying symmetrically on the unit circle. For large positive $\beta$, the steepest descent path passing through the saddle point $k_{+}$is simply the ray $\Gamma$ starting from the origin and passing through $k_{+}$. We note that the steepest descent path is defined by requiring $\operatorname{Im} \varphi(k)=\operatorname{Im} \varphi\left(k_{+}\right)$and $\operatorname{Re} \varphi(k)$ to decrease as $k$ goes away from the saddle; $c f$. [8-10] for the definition and for basic background of the method of steepest descents. Also the steepest ascent path through $k_{+}$is the unit circle, and it ends at the other saddle $k_{-}$; compare Figure 1 for the paths.

Using Cauchy's integral theorem, the integration path $l$ can be deformed to the steepest descent path $\Gamma$. Recalling that the argument $\theta$ may range over $(0, \pi)$ and that the integrand in (3.1) has a simple pole at $k=1$, we divide our discussion into three cases, namely (i) $0<$ $\theta<\pi / 2$, (ii) $\theta=\pi / 2$, and (iii) $\frac{\pi}{2}<\theta<\pi$. We deform the paths case by case.

Case (i). When $0<\theta<\pi / 2$, the steepest descent path passing through $k_{+}$lies in the first quadrant of the complex $k$-plane. We simply deform $l$ into $\Gamma$, and we have

$$
I(\beta)=\frac{D}{2 \pi i} \int_{\Gamma} e^{i \beta\left[k(z+A)-\frac{1}{k} \overline{(z+A)}\right]} \frac{k^{2}+1}{k\left(k^{2}-1\right)} d k .
$$

Case (ii). When $\theta=\pi / 2$, the steepest descent path passing through $k_{+}=1$ coincides with the positive half real axis of the complex $k$-plane. Also $k=1$ is a simple pole of the integrand. The seemingly complicated situation turns out to be easily handled, since $I(\beta)$ can be explicitly given in this case:

$$
I(\beta)=\frac{D}{2 \pi i} \int_{\widetilde{\Gamma}} e^{-\beta y\left(k+\frac{1}{k}\right)} \frac{k^{2}+1}{k\left(k^{2}-1\right)} d k=-\frac{D}{2} e^{-2 y \beta},
$$


Figure 2 The regions I, II, and III in the complex half $z$-plane.

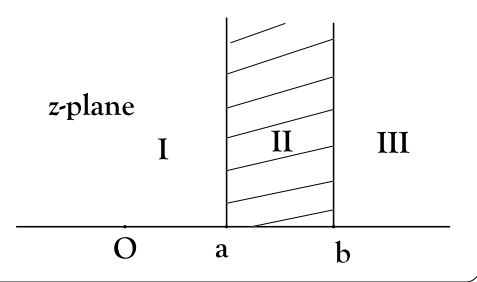

where the integration path $\widetilde{\Gamma}$ consists of two segments $[0,1-\varepsilon]$ and $[1+\varepsilon, \infty)$ and a upper half circle joining them, as illustrated in Figure 1 . The last equality is obtained by using the symmetry of the integral under the transformation $k \leftrightarrow 1 / k$, picking up half of the residue of the integrand at $k=1$, and taking the limit as $\varepsilon \rightarrow 0$.

Case (iii). When $\pi / 2<\theta<\pi$, the steepest descent path $\Gamma$ emanating from the saddle point $k_{+}$is in the fourth quadrant of the complex $k$-plane. To deform the original path $l$ to $\Gamma$, one has to pick up the residue from the simple pole at $k=1$. Accordingly, we have

$$
I(\beta)=\frac{D}{2} e^{-2 y \beta}+\frac{D}{2 \pi i} \int_{\Gamma} e^{i \beta\left[k(z+A)-\frac{1}{k} \overline{(z+A)}\right]} \frac{k^{2}+1}{k\left(k^{2}-1\right)} d k .
$$

We are now in a position to derive the asymptotic approximation for $q(x, y)$ in (3.1) for fixed $z=x+i y \in \Omega$. We denote

$$
z-a=r_{1} e^{i \theta_{1}}, \quad z-b=r_{2} e^{i \theta_{2}} .
$$

Obviously we have $0<\theta_{1}<\theta_{2}<\pi$. We divide the half $z$-plane $\Omega$ into three regions, namely, I, II, and III, defined by $\operatorname{Re} z \in(-\infty, a), \operatorname{Re} z \in(a, b)$ and $\operatorname{Re} z \in(b, \infty)$, respectively; $c f$. Figure 2. Then it is clear that when $z \in \mathrm{III}, \theta_{j} \in(0, \pi / 2)$ for $j=1$, 2; for $z \in \mathrm{II}, \theta_{2} \in(\pi / 2, \pi)$ and $\theta_{1} \in(0, \pi / 2)$; and for $z \in \mathrm{I}, \theta_{1}, \theta_{2} \in(\pi / 2, \pi)$. On the boundaries, when $\operatorname{Re} z=b, \theta_{2}=$ $\pi / 2$, while $\theta_{1}=\pi / 2$ as $\operatorname{Re} z=a$.

Now what remains is just a straightforward application of the method of steepest descents ( $c f .[8-10])$. To be precise, we employ the standard argument of Watson's lemma. Firstly, we introduce

$$
\tau:=\varphi\left(k_{+}\right)-\varphi(k)=-2 r-i r\left(k e^{i \theta}-\frac{1}{k} e^{-i \theta}\right)=r\left(|k|-2+\frac{1}{|k|}\right),
$$

where $k=|k| i e^{-i \theta}$ for $k \in \Gamma$. Obviously $\tau \in[0, \infty)$ on the steepest descent path, and for each $\tau \in(0, \infty)$ there is a pair of points on $\Gamma$, say, $k_{1}$ and $k_{2}$, satisfying (3.7). Indeed, we may specify

$$
\begin{aligned}
& k_{1}=i e^{-i \theta}\left[1+\frac{\tau}{2 r}-\sqrt{\left(1+\frac{\tau}{2 r}\right)^{2}-1}\right] \\
& k_{2}=i e^{-i \theta}\left[1+\frac{\tau}{2 r}+\sqrt{\left(1+\frac{\tau}{2 r}\right)^{2}-1}\right] .
\end{aligned}
$$

Accordingly one obtains

$$
\frac{D}{2 \pi i} \int_{\Gamma} e^{\beta \varphi(k)} \frac{k^{2}+1}{k\left(k^{2}-1\right)} d k=\frac{D}{2 \pi i} e^{-2 r \beta} \int_{0}^{\infty} \Phi(\tau) e^{-\beta \tau} d \tau,
$$


where

$$
\Phi(\tau)=\frac{k_{2}^{2}+1}{k_{2}\left(k_{2}^{2}-1\right)} \frac{d k_{2}}{d \tau}-\frac{k_{1}^{2}+1}{k_{1}\left(k_{1}^{2}-1\right)} \frac{d k_{1}}{d \tau},
$$

which can be expanded into a convergent series for small $\tau$ in the form

$$
\Phi(\tau)=\tau^{-1 / 2} \sum_{l=0}^{\infty} c_{l} \tau^{l}, \quad \tau \in(0, \delta r)
$$

where $\delta$ is a positive constant, and the coefficients $c_{l}=c_{l}(r, \theta)$ can be evaluated in view of (3.8). Waston's lemma implies that the whole contribution to the asymptotic behavior of the integrals in (3.9) comes from the saddle $k=k_{+}$. Substituting (3.10) into (3.9) yields the full asymptotic expansion

$$
\frac{D}{2 \pi i} \int_{\Gamma} e^{\beta \varphi(k)} \frac{k^{2}+1}{k\left(k^{2}-1\right)} d k \sim \frac{D}{2 \pi i} e^{-2 r \beta} \sum_{l=0}^{\infty} \frac{c_{l} \Gamma(l+1 / 2)}{\beta^{l+1 / 2}}, \quad \beta \rightarrow+\infty
$$

where $\Gamma(t)$ is the usual Gamma function. We summarize our discussion as follows.

Theorem 1 For large positive $\beta$ (i.e., high frequencies and, equivalently, large wavenumbers), the following asymptotic expansions of the solution $q(x, y)$ to the boundary value problem (1.1), (1.3) of the modified Helmholtz equation hold:

$$
\begin{aligned}
q(x, y) \sim & \frac{D}{2 \pi i} \sum_{j=1}^{2}(-1)^{j+1} e^{-2 r_{j} \beta} \sum_{l=0}^{\infty} \frac{c_{l}\left(r_{j}, \theta_{j}\right) \Gamma(l+1 / 2)}{\beta^{l+1 / 2}} \\
& +\frac{D}{2} e^{-2 y \beta} \cdot \begin{cases}0, & z \in \mathrm{I} \cup \mathrm{III} ; \\
1, & z \in \mathrm{II}\end{cases}
\end{aligned}
$$

as $\beta \rightarrow+\infty$, where $r_{j}$ and $\theta_{j}$ are defined in (3.6). Two exceptional cases are

$$
q(x, y) \sim-\frac{D}{2 \pi i} e^{-2 r_{2} \beta} \sum_{l=0}^{\infty} \frac{c_{l}\left(r_{2}, \theta_{2}\right) \Gamma(l+1 / 2)}{\beta^{l+1 / 2}}+\frac{D}{4} e^{-2 y \beta},
$$

as $\beta \rightarrow+\infty$ and $\operatorname{Re} z=a$, and

$$
q(x, y) \sim \frac{D}{2 \pi i} e^{-2 r_{1} \beta} \sum_{l=0}^{\infty} \frac{c_{l}\left(r_{j}, \theta_{1}\right) \Gamma(l+1 / 2)}{\beta^{l+1 / 2}}+\frac{D}{4} e^{-2 y \beta},
$$

as $\beta \rightarrow+\infty$ and $\operatorname{Re} z=b$. 


\section{References}

1. Chandler-Wilde, SN, Graham, IG: Boundary integral methods in high-frequency scattering. In: Engquist, B, Fokas, T, Hairer, E, Iserles, A (eds.) Highly Oscillatory Problems, pp. 154-193. Cambridge University Press, Cambridge (2009)

2. Arden, S, Chandler-Wilde, SN, Langdon, S: A collocation method for high frequency scattering by convex polygons. J. Comput. Appl. Math. 204, 334-343 (2007)

3. Bao, G, Wei, G-W, Zhao, S: Numerical solution of the Helmholtz equation with high wavenumbers. Int. J. Numer. Methods Eng. 59, 389-408 (2004)

4. Chandler-Wilde, SN, Langdon, S: A Galerkin boundary element method for high frequency scattering by convex polygons. SIAM J. Numer. Anal. 45, 610-640 (2007)

5. Kim, S, Shin, C-S, Keller, JB: High-frequency asymptotics for the numerical solution of the Helmholtz equation. Appl. Math. Lett. 18, 797-804 (2005)

6. Langdon, S, Chandler-Wilde, SN: A wavenumber independent boundary element method for acoustic scattering problem. SIAM J. Numer. Anal. 43, 2450-2477 (2006)

7. Keller, JB: Progress and prospects in the theory of linear wave propagation. SIAM Rev. 21, 229 (1979)

8. Copson, ET: Asymptotic Expansions. Cambridge Tracts in Math. and Math. Phys., vol. 55. Cambridge University Press, London (1965)

9. Olver, FWJ: Asymptotics and Special Functions. Academic Press, New York (1974)

10. Wong, R: Asymptotic Approximations of Integrals. Academic Press, Boston (1989)

11. Fokas, AS: A Unified Approach to Boundary Value Problems. SIAM, Philadelphia (2008)

12. Spence, EA: Boundary Value Problems for Linear Elliptic PDEs. PhD thesis, University of Cambridge (2010)

10.1186/1687-2770-2014-45

Cite this article as: Huang: High-frequency asymptotics for the modified Helmholtz equation in a half-plane.

Boundary Value Problems 2014, 2014:45

\section{Submit your manuscript to a SpringerOpen ${ }^{0}$ journal and benefit from:}

- Convenient online submission

- Rigorous peer review

- Immediate publication on acceptance

- Open access: articles freely available online

- High visibility within the field

- Retaining the copyright to your article 